\title{
Zimmermann, Thomas (2016): Das Unbehagen in der Planung. Hochwassergefahren, Regionalplanung und ihre Wirkungen
}

\author{
Lemgo: Verlag Dorothea Rohn. 316 S., 5 Abb., 5 Tab.
}

\section{Sylvia Kruse ${ }^{1}$}

Online publiziert: 20. Juli 2017

(c) Springer-Verlag GmbH Deutschland 2017

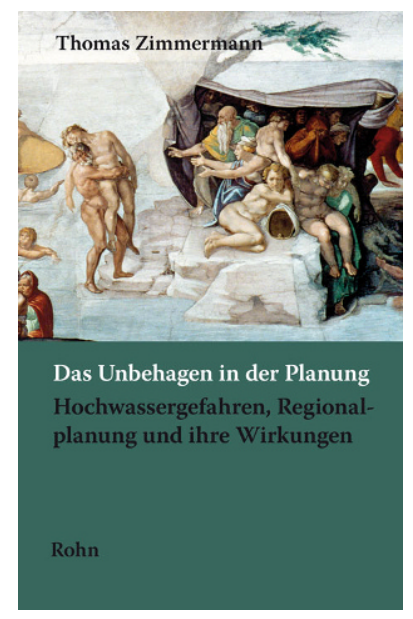

\section{Traumatisierte Regionalplanung? Zum Verhält- nis von vorbeugendem Hochwasserschutz, Regionalplanung und ihrer Wirkung}

Ist die Regionalplanung traumatisiert? Ein Trauma, so führt Thomas Zimmermann in dem hier zu besprechenden Buch „Das Unbehagen in der Planung. Hochwassergefahren, Regionalplanung und ihre Wirkungen“ mit Bezug zu Sigmund Freud aus, ist ein Ereignis im Leben eines Subjektes, das das Subjekt als unfähig erscheinen lässt, es in der Folge in seiner Aktivität hemmt und sogar - im Fall traumatischer Neurosen - zu einem Wiederholungszwang führen

Dr. Sylvia Kruse

sylvia.kruse@ifp.uni-freiburg.de

1 Professur für Forst- und Umweltpolitik, Albert-Ludwigs-Universität Freiburg, Tennenbacher Straße 4, 79106 Freiburg, Deutschland kann (S. 268 ff.). Nun ist die Regionalplanung kein Subjekt im Sinne Freuds. Dennoch überträgt Zimmermann diese psychoanalytische Perspektive auf die Regionalplanung und auf Gesellschaften, die wiederholt Katastrophenerfahrungen ausgesetzt sind und dennoch (oder gerade deswegen) überschwemmungsgefährdete Bereiche nicht verlassen, sondern immer wieder erneut besiedeln.

Mit der Übertragung der freudianisch-lacanianischen Perspektive auf die Fragen der Wirksamkeit von Regionalplanung und der Kombination dieser Perspektive mit klassischer policy-orientierter Evaluationsforschung irritiert und inspiriert Zimmermann. Wie geht der Mensch mit Gefahren um? Wie begegnet er der Natur? Welche Rolle spielt dabei die räumliche Planung, eine sich der Vorsorge und der Nachhaltigkeit verpflichtende Disziplin? Wie wirken die bisherigen planerischen Grundsätze im Handlungsfeld des vorbeugenden Hochwasserschutzes? Gerade angesichts des im November 2016 vorgelegten Gesetzentwurfes zur Novellierung des Hochwasserschutzgesetzes ist es an der Zeit, sich erneut mit der Rolle und Wirksamkeit der räumlichen Planung und dem Verhältnis zwischen Mensch und Natur im Umgang mit Hochwasserereignissen Gedanken zu machen. Dieser Aufgabe stellt sich Zimmermann in seiner Dissertation, die er 2016 im Verlag Dorothea Rohn als Buch veröffentlichte.

Der Titel „Das Unbehagen in der Planung“ ist als Analogie zur von Freud verfassten Schrift „,Das Unbehagen in der Kultur“ gewählt und verweist damit auf den freudianisch-lacanianischen Zugang in seiner Übertragung auf die Raumplanung durch Gunder und Hillier (2009), den Zimmermann neben einer planungswissenschaftlichen Evaluation von Regionalplänen auf seinen Forschungsgegenstand anwendet. Diese mitunter irritierende, mindestens aber ungewöhnliche Kombination theoretischer Zugänge zu der Frage nach den Wirkungen von regionalplanerischem Han- 
deln auf den vorbeugenden Hochwasserschutz fordern die Leser heraus, den Forschungsgegenstand immer wieder aus zwei sehr unterschiedlichen Perspektiven heraus zu betrachten und zu reflektieren. Entlang einer Programmtheorie, die Zimmermann durch Elemente der akteurorientierten PolicyForschung ergänzt, untersucht er die Wirkung planerischer Grundsätze zum vorbeugenden Hochwasserschutz. Dazu analysiert er den Vollzug der Rahmenvorgaben des Bundes durch die Regionalplanung, indem er Inhalte von $24 \mathrm{Re}-$ gionalplänen aus Deutschland mit einer Clusteranalyse untersucht, und identifiziert verschiedene Steuerungsansätze zum vorbeugenden Hochwasserschutz. In zwei Fallstudien „Region Oberes Elbtal/Osterzgebirge“ und „Region Mittelthüringen“ untersucht er anschließend in einer vertieften Analyse die Inhalte der bestehenden Regionalpläne, wofür er mittels Experteninterviews und Dokumentenauswertung Daten für die Wirkungsanalyse sammelt. Diese werden entlang einer durch das ,Institutional Analysis and Development Framework“ nach Ostrom (2005) ergänzten Programmtheorie evaluiert, wobei der Fokus neben der Wirkungsanalyse auf Entscheidungsprozessen und Interaktionen zwischen den Akteuren in den beiden Fallstudien liegt. Die Arbeit schließt mit sechs Thesen, in denen Zimmermann die gesellschaftliche Bedeutung der Verminderung von Hochwassergefahren und die Wirkung des regionalplanerischen Instrumentariums hinterfragt und eine Neuausrichtung der Regionalplanung skizziert. Die regionalplanerischen Instrumente kommen dabei nicht gerade gut weg. Zimmermann attestiert ihnen, dass mit ihnen die intendierte Wirkung, nämlich die Verringerung von Hochwasserentstehung und -gefahren, nicht erreicht werden kann und ihnen sogar „das kollektive Begehren“, also der Wille, dazu fehlen würde (S. 275). Vielmehr solle - und hier bleibt Zimmermann unbestimmt, wer die Verantwortung dafür tragen sollte - ein Verständnis dafür entwickelt werden, dass eine vollständige Beherrschbarkeit von Naturgefahren unmöglich ist. Diese Forderung ist allerdings nicht neu, sondern wird von Kritikern des Machbarkeitsideals staatlicher Planung bereits seit dem enttäuschten Planungsoptimismus und dem proklamierten Scheitern des regulativen Planungsanspruches der 1980er-Jahre nicht nur, aber auch in Bezug auf den planerischen Umgang mit Naturgefahren diskutiert.

Zwar kreativ, aber nicht unproblematisch, ist die Kombination zweier sehr unterschiedlicher Ansätze: Zum einen führt Zimmermann eine klassische Wirkungsanalyse durch, die Reichweite und Wirkung von planerischem Handeln entlang von verschiedenen Struktur- und Prozesskategorien beurteilt und erkenntnistheoretisch einem modernen Paradigma folgt. Zum anderen verfolgt er einen psychoanalytischen Ansatz, der häufig als nichtwissenschaftlich kritisiert wird und in seiner Weiterführung durch Lacan dem Poststrukturalismus und damit einem postmodernen Paradigma zugeordnet werden kann. Die Kompatibilität und mögliche Unvereinbarkeiten dieser beiden Ansätze hätte Zimmermann durchaus offensiver ansprechen und reflektieren können, um deutlich zu machen, warum es sinnvoll ist, gerade diese beiden Ansätze zu verbinden, und wo jeweils die Grenzen der Analyse liegen. Nichtsdestotrotz eröffnen sich gerade durch diese ungewöhnliche Kombination von Forschungsperspektiven den Lesern immer wieder neue, teilweise analytische, teilweise assoziative Zusammenhänge, die durchaus helfen können, die eingefahrenen Strukturen der Planung neu zu denken.

Bezüglich seiner Forderungen an die Neuausrichtung der Regionalplanung hätte Zimmermann allerdings noch präziser argumentieren können: Wen sieht er in der Pflicht, wenn er verlangt, dass die Regionalplanung verstärkt durchsetzungsstarke Ziele der Raumordnung entwickeln und umsetzen solle? Meint er hier den Gesetzgeber und die Planungsbehörden auf der Ebene der Bundesländer? Oder sollte diese Neuausrichtung durch eine veränderte Planungspraxis auf der Ebene der regionalen Akteure geschehen und durch wen genau? Wann sind Ziele der Raumordnung eigentlich durchsetzungsstark und vor welchem normativen Hintergrund kann dies bewertet werden? Diese Aufforderungen zur Präzisierung zeigen, dass die Debatte um eine Neuausrichtung der Regionalplanung durch die empirische und theoretische Arbeit Zimmermanns zwar lange noch nicht abgeschlossen ist, durchaus aber durch seine theoretischen und empirischen Ausführungen angeregt und weitergebracht wurde. Genau darin liegt neben manchen ungeklärten Irritationen die Stärke des Buches: Es regt zu Reflexion und Perspektivwechsel an.

\section{Literatur}

Gunder, M.; Hillier, J. (2009): Planning in ten words or less: a Lacanian entanglement with spatial planning. Farnham.

Ostrom, E. (2005): Understanding institutional diversity. Princeton. 